\title{
Adjacency Effects of Layered Clouds by Means of Monte Carlo Ray Tracing
}

\author{
Kohei Arai \\ Faculty of Science and Engineering \\ Saga University, Saga City, Japan
}

\begin{abstract}
Adjacency effects from layered box shaped clouds are clarified by means of Monte Carlo Simulation: MCS taking into account a phase function of cloud particles and multilayered plane parallel atmosphere. MCS allows estimation of top of the atmosphere radiance. Influences on adjacency effects of phase function of the clouds in concern and the number of layers of the plane parallel atmosphere are also clarified together with the effects from the top and the bottom clouds. There are 10 of cloud types in meteorological definition. One-layer cloud for cumulus and cumulonimbus clouds are investigated in this study.
\end{abstract}

Keywords-Monte Carlo simulation; top of the atmosphere radiance; cloud type; adjacency effects; layered clouds

\section{INTRODUCTION}

As described in [1], [2] the adjacent effect is a phenomenon in which reflected light components from other than the observation target are superimposed on the luminance from the target. For example, the brightness of an object with a small emission brightness, such as the sea surface, may be affected by several times the effect of cloud adjacency. Correction of this effect is important. According to Wilson A [3], when Turbid Case 2 seawater1 is targeted, the sea surface emission brightness in the visible wavelength range is $2-3.5[\mu \mathrm{W} / \mathrm{cm} /$ str / nm], which is about 100 times that of high-rise clouds. It also extends. Therefore, the $1 \%$ adjacent effect is comparable to the sea surface emission brightness and cannot be ignored.

There are reports about the cloud adjacent effect by Monte Carlo method ${ }^{2}$ already [4]. These are Monte Carlo simulations analysis of radiative transfer analytically assuming the ground surface, sea level, clouds, etc. in various atmospheric conditions

This Monte Carlo method was applied when it was impossible. In past studies, the effect of multiple reflections between the cloud base and the ground surface, the effect of scattering in clouds with different optical thicknesses, and the effect of multiple reflections between the cloud side and the ground surface were clarified by the Monte Carlo method. Clouds are layered. The effect of each layer on the peripheral effect of clouds is also clarified [5]. However, in these simulations, in order to shorten the time required for the Monte Carlo simulation, the atmosphere and clouds are represented by

1 https://www.researchgate.net/publication/232712020_Optical_ Modeling_of_Ocean_Waters_Is_the_Case_1_Case_2_Classification_Still_Us eful.

${ }^{2}$ https://virial.com/monte-carlo-ray-tracing.html. a one-layer model, taking into account the vertical nonuniformity of the real atmosphere and clouds.

The level of cloud droplets replacing the atmosphere with a multilayer parallel plate model [6].

The evaluation result of the cloud adjacency effect by Monte Carlo simulation using the same correlation number as the aerosol phase function has already been reported [7]. However, cloud distribution and cloud particle phase function were not considered. The phase function of cloud particles is the same as that of aerosol [8]. On the other hand, adjacency effect of layered clouds estimated with Monte-Carlo simulation is proposed and validated [9].

Evaluation of cirrus cloud detection accuracy of GOSAT/CAI and Landsat-8 with Laser Radar: Lidar is proposed and is validated with Calipso data [10]. In the meantime, comparative study on cloud parameter estimation among GOSAT/CAI, MODIS, CALIPSO/CALIOP and Landsat-8/OLI has been done with laser radar as truth data [11]. Meanwhile, thresholding based method for rain, cloud detection with NOAA/AVHRR data by means of Jacobi iteration method is proposed [12].

Typically, Radiative Transfer Equation: RTE has to be solved for the situations of which targeted scene includes clouds. It, however, is difficult to formulate RTE for such situation of remote sensing satellite data in particular for layered clouds. In this paper, a method for solving RTE for such situation (Layered clouds are included in the targeted scene of remote sensing satellite images by means of Monte Carlo Ray Tracing: MCRT.

The following section describes layered clouds in concern followed by Monte Carlo simulation (MCRT) in the presence of clouds in a uniform atmosphere. Then some results from simulation study is described followed by conclusion with some discussions.

\section{LAYERED CLOUDS IN CONCERN}

When the cloud base height and the cloud top height are $\mathrm{Ht}$ and $\mathrm{Hb}$, respectively, the 10 cloud types [9] defined by the World Meteorological Organization are as shown in Table I. In this study,

- One-layer cloud for cumulus and cumulonimbus clouds 
- Adjacent effects are evaluated for two-layered clouds, which are layered clouds such as lower-layer turbulent clouds, stratocumulus clouds, and middle-layer highcumulus clouds and high-rise clouds. Fig. 1 schematically shows the one layer and two layer clouds assumed here.

\section{A. One Layer Clouds}

Cumulus clouds and cumulonimbus clouds, which are the targets of one layer of clouds, are clouds that develop vertically and are sometimes called convective clouds. Cumulus clouds can spread from near the ground to the middle and upper layers, and are often seen in the daytime when the weather is nice. The cumulonimbus is a dense cloud that grows vertically, and lightning and heavy rain fall from this cloud. Such lower clouds are considered to have a strong reflection effect that reduces sunlight by reflecting sunlight and reaching the ground surface. According to GLAS model [13], the albedo of cumulonimbus $(\mathrm{Cb})$ is 0.8 and the albedo of cumulus $(\mathrm{Cu})$ is 0.7. Therefore, this time, from the GLAS model of [13], the reflectance $(\mathrm{R})$ of the cloud of one layer is 0.7 and the optical thickness (OD) is 9.0.

\section{B. Two Layer Clouds}

The high cumulus clouds that are the target of the upper part of the two-layer clouds are white or gray, rounded or rolled, and the high clouds are grayish or have a uniform layer with a pale bluish color. Turbulent clouds in the lower layer are dark gray thick clouds that extend from low to high, completely covering the sun and moon and making it rain.

TABLE. I. 10 CLOUD TYPES DEFINED BY THE WORLD METEOROLOGICAL ORGANIZATION

\begin{tabular}{|l|l|l|}
\hline Name & Cloud bottom height $(\mathrm{km})$ & Cloud top height $(\mathrm{km})$ \\
\hline Cirrus & 6 & \\
\hline Cirrocumulus & 6 & \\
\hline Cirrostratus & 6 & \\
\hline Altocumulus & 2 & 6 \\
\hline Altostratus & 2 & 6 \\
\hline Nimbostratus & 0 & 2 \\
\hline Stratocumulus & 0 & 2 \\
\hline Stratus & 0 & 2 \\
\hline Cumulus & 0.5 & 6 \\
\hline Cumulonimbus & 0.5 & 6 \\
\hline
\end{tabular}

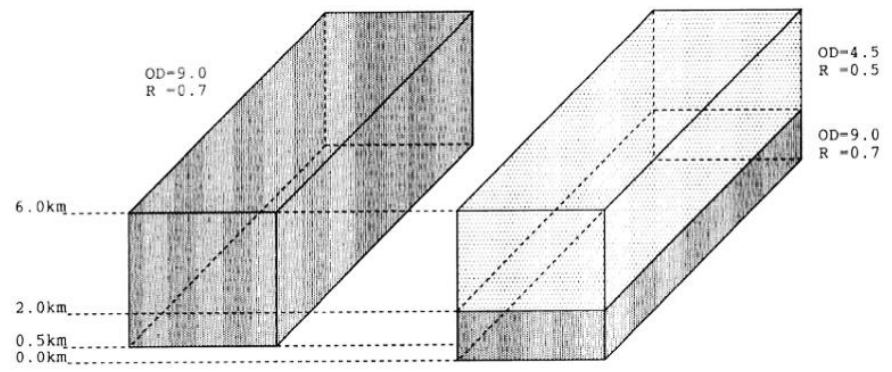

Fig. 1. Assumed One and Two Layer Clouds.
Cumulus clouds are clouds that appear about $2000 \mathrm{~m}$ from the ground, and are layered clouds such as gray or whitish or grayish white clusters or thin plates. The stratus has a uniform cloud bottom and drizzle with gray clouds. Such middle-layer clouds are optically thinner than the lower-layer clouds and pass solar radiation well. According to the GLAS model, the albedo of the high clouds (As) is 0.5 and the albedo of the stratus St is 0.7. Therefore, also from the GLAS model of [13], the upper cloud reflectance $(\mathrm{R})$ is 0.5 , the optical thickness $(\mathrm{OD})$ is 4.5 , the lower cloud reflectance (R) is 0.7 , and the optical thickness. (OD) was set to 9.0.

\section{Monte Carlo Simulation in the PRESENCE OF Clouds In A UNIFORM ATMOSPHERE}

\section{A. Simulation Outline}

Numerically solving the problems of multiple scattering and reflection of light in the atmospheric and surface systems close to reality is considered by the Monte Carlo method. As shown in Fig. 2, assuming a three-dimensional atmospheric cell with an isolated cloud, photons are randomly generated from the upper surface of the atmosphere and incident so as to follow the solar incident direction (zenith angle and azimuth) and $\left(\mu_{0}, \varphi_{0}\right)$. Then, the travel distance of each generated photon and its scattering and absorption with atmospheric particles are tracked with probabilistic determination according to the atmospheric optical characteristic parameters.

In this simulation, the radiance emitted from the upper surface of the atmosphere is calculated by counting the number of photons emitted from the upper surface of the atmospheric cell.

Here, the following assumptions are made.

When a photon leaves the range of the atmospheric cell due to scattering, the photon enters the atmospheric cell from a surface opposite to the surface.

The atmosphere is optically uniform within the same layer (uniform in the horizontal direction and non-uniform in the vertical direction).

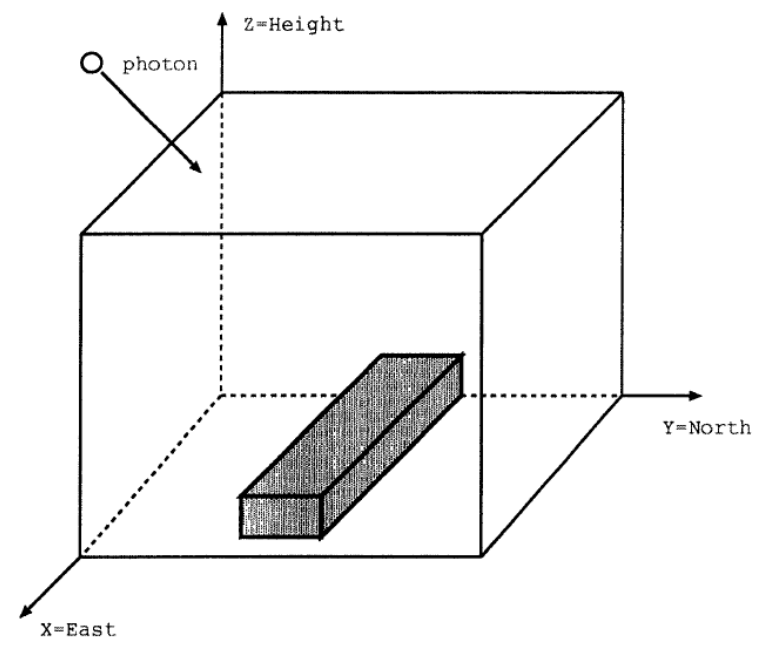

Fig. 2. Assumed a Three-Dimensional Atmospheric Cell with an Isolated Cloud. 
The ground surface is a Lambertian surface that is flat and has a uniform reflectivity.

A photon enters the atmospheric cell according to the incident angle of the sun. At this time, the distance (free path) that the photon travels in the atmosphere is determined by using a random number, and it is determined according to their optical thickness whether the particle that the photon collides next is an air molecule or an aerosol particle. Determine whether the photon is absorbed or scattered by the single scattering albedo of the particle, and if it is scattered, determine the scattering angle by the phase function of the particle and also calculate the free path.

These are repeated, and photons propagate in the atmosphere. The surface of the cloud was assumed to be a Lambertian surface ${ }^{3}$, reflected according to its reflectivity, and transmitted with the transmittance minus the reflectivity. Furthermore, assuming that the ground surface is also a Lambertian surface, reflection was performed according to the reflectivity, and photons were absorbed with the absorptivity subtracted from 1.

Real clouds and the ground surface are thought to be surfaces with anisotropic two-way reflection characteristics such as Minnaert's law ${ }^{4}$, but in particular, there was no paper mentioning reflection characteristics such as the minerality coefficient of clouds. Here, the assumption of Lambert property was also made from the ease of calculation. For photons that jump out of the simulation cell, the photons are incident in the same direction from the position opposite to the position where they jump out. The details of these Monte Carlo methods are described in [5].

In the Monte Carlo method, the number of photons incident on the atmospheric cell needs to be sufficiently large. When this is small, the variation of the solution is large and the simulation is in a state where the signal-to-noise ratio is bad. As an example, calculating the radiance 1 at the top of the atmosphere while changing the number of photons gives Fig. 3 . This shows that about 1 billion photons are required to obtain stable atmospheric top radiance. Therefore, the number of photons used in the subsequent Monte Carlo method was set to 1 billion.

\section{B. Simulation Parameters}

The setting of the Monte Carlo simulation parameters was the same as in [5]. That is, the size of the atmospheric cell was $50 \mathrm{~km}$ from east to west, $50 \mathrm{~km}$ from north to south, and $50 \mathrm{~km}$ high. The atmospheric composition was only air molecules and aerosols, and those contained in the extra-atmospheric solar radiant flux (MODTRAN ${ }^{5}-3.7[14]$ were used.

The real part of the complex refractive index ${ }^{6}$ of the aerosol was 1.44 , the imaginary part was -0.005 , and the single scattering albedo ${ }^{7}$ was 0.9318 . In addition, the aerosol particle

\footnotetext{
${ }^{3}$ https://en.wikipedia.org/wiki/Lambertian_reflectance

${ }^{4} \mathrm{https}: / /$ www.researchgate.net/publication/228399602_Comparison _of_the_Minnaert_constant_for_different_forest_types_using_multitemporal_SPOTHRV_data

${ }_{6}^{5} \mathrm{http}: / /$ modtran.spectral.com/

${ }^{6} \mathrm{http} / / /$ eodg.atm.ox.ac.uk/ARIA/

${ }^{7}$ https://en.wikipedia.org/wiki/Single-scattering_albedo
}

size distribution is the Junge distribution ${ }^{8}$, the Junge parameter is 3 , the wavelength is $0.5 \mu \mathrm{m}$, the solar zenith angle is 30 degrees, the solar azimuth angle is 120 degrees from north to west, and the ground surface is uniform on the Lambert surface. When sunlight is incident from the 180 degree direction, the adjacent effect is the largest, and the incidence from the 90 degree direction is the smallest.

The quantitative relationship between the incident condition of sunlight and the adjacent effect will be described in another opportunity. Here, the case of 120 degree incidence is taken up as an example. In addition, the satellite altitude can be set arbitrarily, but here we assume the $500 \mathrm{~km}$ adopted by the JERS- $1^{9}$ satellite, etc. and the instantaneous visual field is assumed to be $700 \mathrm{~m} \times 700 \mathrm{~m}$ on the ground surface. That is, the situation where a push bloom type radiometer with an instantaneous field of view of about 0.0014 radians $=0.8$ degrees is mounted on the satellite is simulated. A bin of approximately 0.8 degrees $\times 0.8$ degrees was set at the top of the atmosphere at $50 \mathrm{~km}$ altitude, and the number of photons entering the bin within the instantaneous viewing angle was counted.

The bins were arranged in the $x$ direction (east-west) and scanned in the y direction (north-south) to simulate a push bloom. As shown in Fig. 4, the cloud was a rectangular parallelepiped $(10 \mathrm{~km}$ in the $\mathrm{x}$ direction, $50 \mathrm{~km}$ in the $\mathrm{y}$ direction) and placed in the center of the atmospheric cell. The cloud surface was a Lambertian surface. The ground surface other than this cloud assumes a flat sea surface.

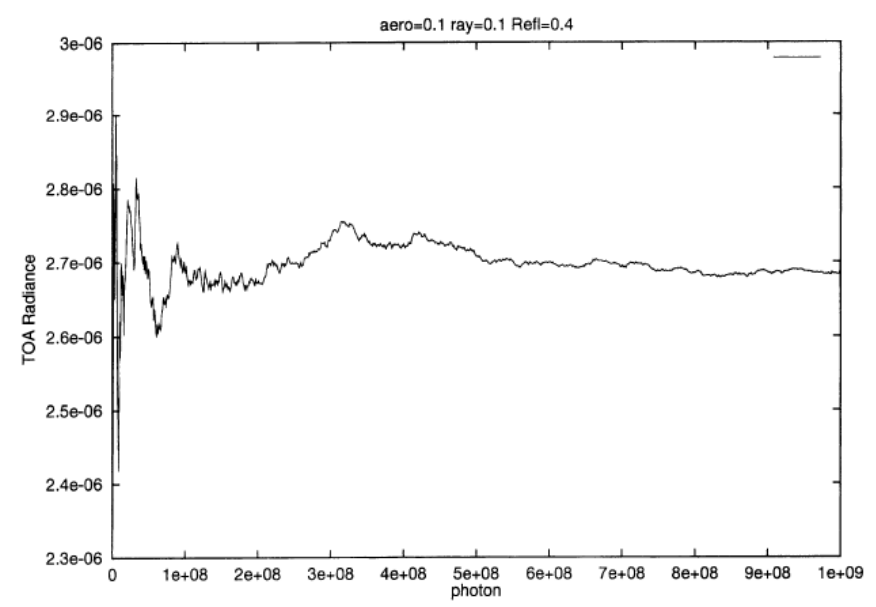

Fig. 3. A Relationship between the Top of the Atmosphere (ToA) Radiance and the Number of Photons ${ }^{10}$ aero : Aerosol optical Depth, ray: Rayleigh Optical Depth, Refl : Reflectance.

\section{Adjacency Effect}

Differences in the adjacent effects of the one layer and the two layer clouds comes as evaluation items of the adjacent effects. Effects of the two-layer clouds on the adjacent effects

\footnotetext{
${ }^{8} \mathrm{https}: / /$ sites.google.com/site/aerosolpedia/yong-yurisuto/guang-sanluan $/ 10$

${ }^{9} \mathrm{https}: / /$ ssl.jspacesystems.or.jp/ersdac/Projects/JERS1/JOPS/JOPS E.html

${ }^{10}$ The radiance when the ground surface reflectance is set to 0.4 , the aerosol optical thickness is set to 0.1 , and the air molecule optical thickness is set to 0.1 .
} 
of the clouds, effects on the side effects of the cloud, and adjacency of the phase function of the cloud particles. The effect on the effect was considered. Author in [5] clarified the difference in the adjacent effect between the first and second clouds, the effect of the two clouds on the adjacent effect by layer, the effect on the adjacent effect on the side of the cloud, etc. The effect of the phase function ${ }^{11}$ of cloud particles is investigated.

In order to evaluate this adjacency effect, the slope factor (D99) defined in [5] is defined again. As shown in Fig. 5, D99 is the distance from the edge of the cloud until the influence of the adjacent effect on the brightness of the cloud gradually decreases until it reaches $1 \%$ of the average sea surface brightness. This $1 \%$ adjacency effect is equivalent to a few levels if the quantization bit number of the satellite-borne sensor is 8 bits, and cannot be ignored. Considering that the maximum input radiance of the onboard sensor is set so as not to saturate even when observing objects with high brightness levels such as clouds and snow and ice, this $1 \%$ adjacent effect is reflected in the sea surface emission brightness. It is comparable and cannot be ignored.

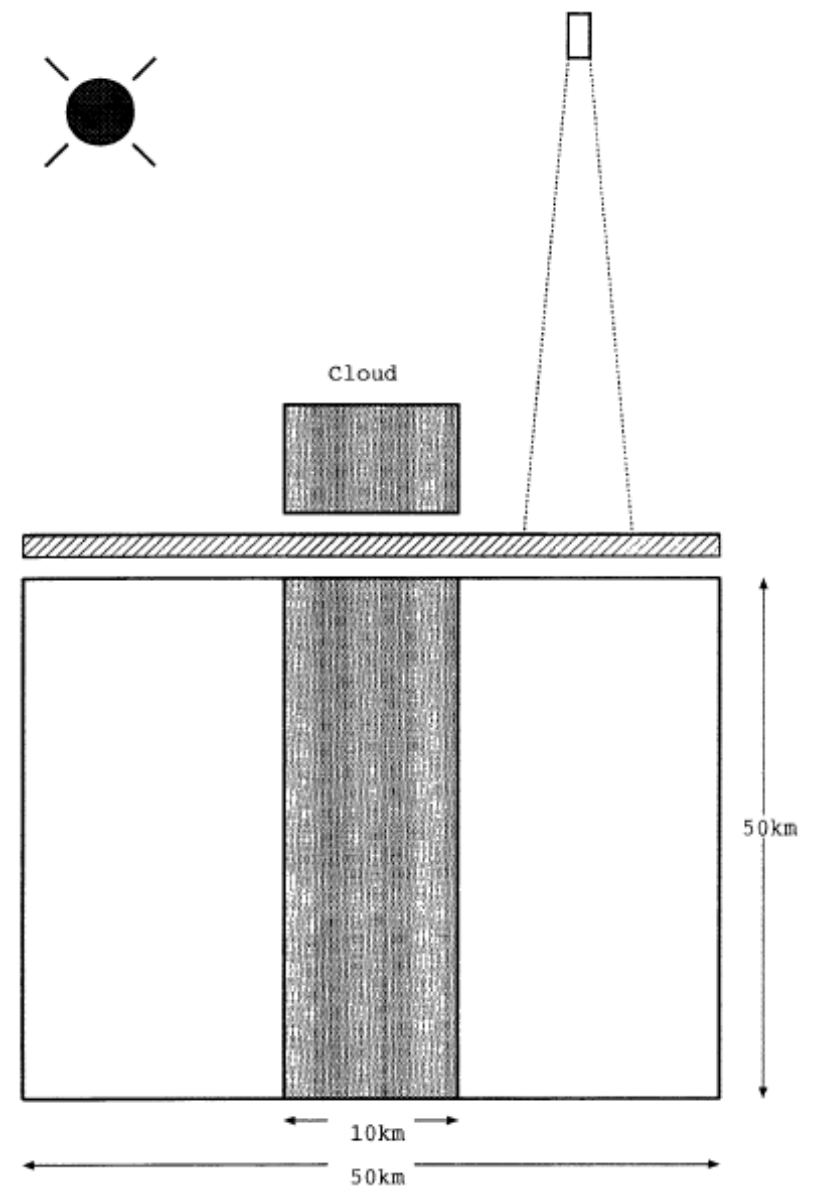

Fig. 4. Rectangular Parallelepiped (10 km in the $\mathrm{x}$ Direction, $50 \mathrm{~km}$ in the $\mathrm{Y}$ Direction) Cloud.

\footnotetext{
${ }^{11}$ https://encyclopedia2.thefreedictionary.com/phase+function
}

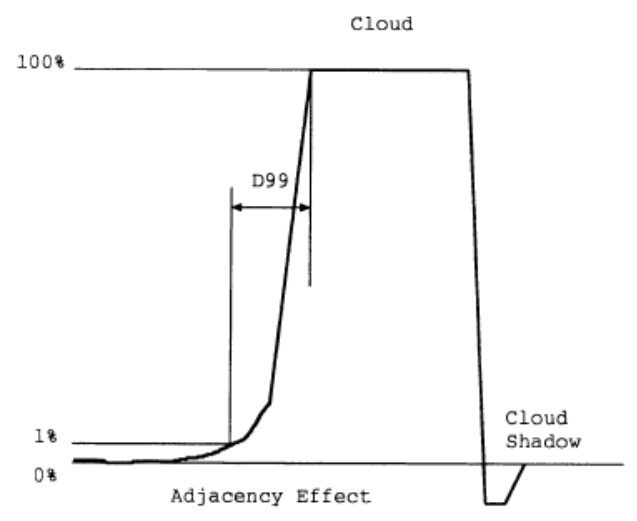

Fig. 5. The Definition of Slope Factor (D99).

\section{EXPERIMENTS}

\section{A. Comparison between One Layer and Two Layer Clouds}

Fig. 6 shows a comparison of phase functions between aerosol and cloud particles calculated with Mie scattering theory while Fig. 7 shows a comparison of the adjacency effect of the single layered cloud with phase functions of aerosol and cloud particle(the unit of Top of the atmosphere: ToA radiance is $\mathrm{W} / \mathrm{cm}^{2} / \mathrm{str} /$ micrometer).

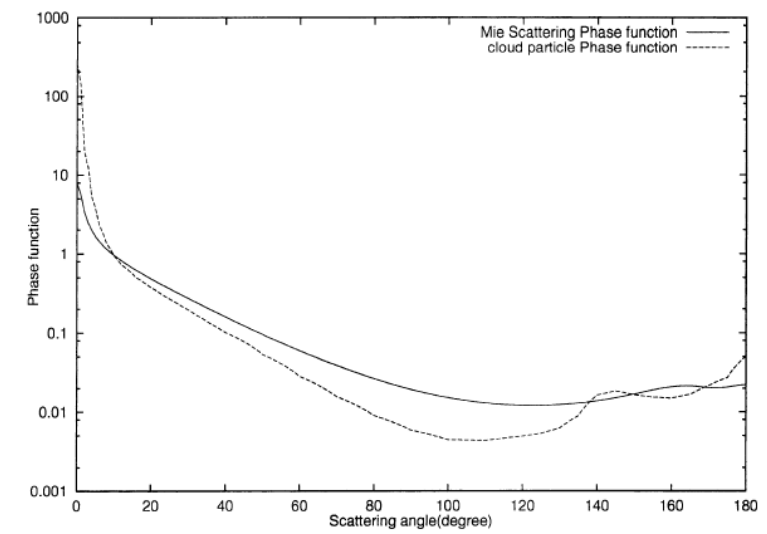

Fig. 6. A Comparison of Phase Functions between Aerosol and Cloud Particles Calculated with Mie Scattering Theory.

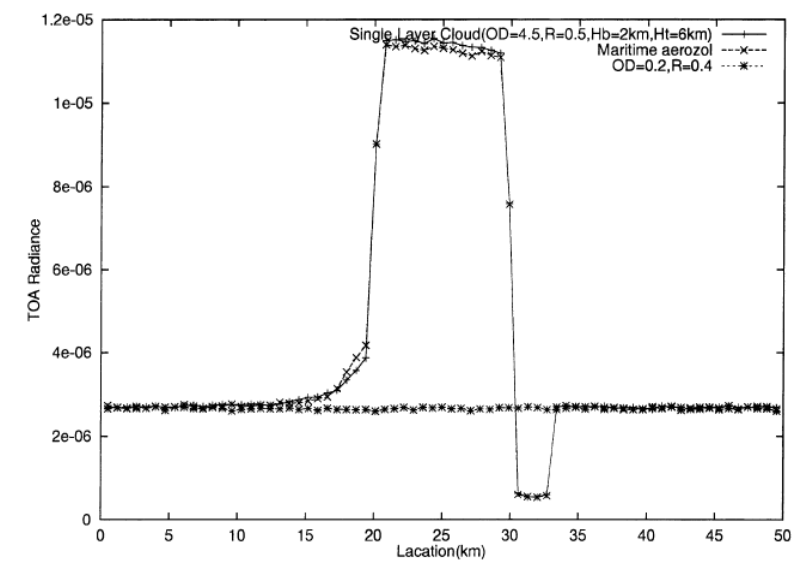

Fig. 7. A Comparison of the Adjacency Effect of the Single Layered Cloud with Phase Functions of Aerosol and Cloud Particle(the unit of ToA radiance is $\left.\mathrm{W} / \mathrm{cm}^{2} / \mathrm{str} / \mathrm{micrometer}\right)$. 
The D99 of the first layer cloud is $4.8 \mathrm{~km}$ and the D99 of the second layer cloud is $4.1 \mathrm{~km}$. The average radiance of the cloud is larger in the one-layer cloud than in the two-layer cloud. This is because the reflectance of the upper cloud of the two-layer cloud is 0.5 , whereas the reflectance of the one-layer cloud is 0.7 , and more reflection occurred on the upper surface of the one-layer cloud. It is done. In addition, because it is set so that sunlight enters from the left zenith angle 30 degrees, a shadow of about $3 \mathrm{~km}$ appears on the right side of the cloud on the opposite side, and the upper edge brightness at this point is rapidly decreasing.

Also, it is possible to show a comparison of the adjacent effects of clouds with the two-layer clouds and the upper clouds removed. The D99 of the two-layer cloud is $4.1 \mathrm{~km}$, while the D99 of the one-layer cloud with the cloud above the two-layer cloud removed is $2.0 \mathrm{~km}$, indicating that the adjacent effect is small.

Moreover, the cloud with the cloud height removed is lower than that of the two-layer cloud with a cloud top height of $2 \mathrm{~km}$, and the cloud shadow is smaller. The cloud with the upper layer removed has a lower cloud top height and smaller volume than the one layer cloud, but the average atmospheric top brightness of the cloud is not much different. This shows that the upper atmospheric brightness of the cloud is dominated by the reflectance of the cloud upper surface.

The D99 of the two-layer cloud is $4.1 \mathrm{~km}$, whereas the D99 of the one-layer cloud with the lower layer of the two-layer cloud removed is $7.6 \mathrm{~km}$, indicating that the adjacent effect is considerably large. This shows that in the adjacent effect of two layers of clouds, the upper layer cloud increases the adjacent effect and the lower layer cloud decreases the adjacent effect. The cloud with the lower clouds removed has a greater adjacency effect despite the optical thickness of 4.5 , reflectance of 0.5 and thin clouds. This is thought to be due to the fact that photons that are transmitted without being reflected by the cloud and scattered within the cloud have a great influence on the adjacent effect.

According to [5], the phase function of the cloud particle is an aerosol in the atmosphere (the complex refractive index is 1.44 for the real part, 0.005 for the imaginary part ${ }^{12}$, and the aerosol distribution is the Junge distribution). The experiment was carried out assuming the same phase function as the Junge parameter 3, the minimum radius of the particle size was 0.01 $\mu \mathrm{m}$, the maximum radius was $10 \mu \mathrm{m}$, and the wavelength was $0.5 \mu \mathrm{m}$. However, the actual cloud particle size has a maximum radius of 15 to $30 \mu \mathrm{m}$ and the largest number is 3 to $7 \mu \mathrm{m}$ [15], which is larger than the aerosol in the atmosphere.

For aerosols, the scattering theory of electromagnetic waves by particles of any size assuming a spherical shape, Mie scattering theory is used. In Mie scattering, when the wavelength of light is the same, the forward scattering becomes more prominent as the particle size of the scattered particles is larger. In this study, an average phase function based on the Mie scattering theory was obtained by considering

12 This is equivalent to the mixture of aerosol derived from sea salt particles (Oceanic) and water-soluble aerosol (Water Soluble) in a ratio of 6: 4 according to the Maxell-Garnet mixing rule. the water soluble particle size of $15-30 \mu \mathrm{m}$ in [15] as the phase function of cloud particles.

In the case of a single-layer cloud, the adjacent effect was compared when the phase function of cloud particles was the same as that of atmospheric aerosol and when the average cloud particle phase function was used. The results are shown in Fig. 7 and Table II. When the phase function of the cloud particle is that of an average cloud particle that increases forward scattering, the brightness near the cloud edge increases, but both the average radiance and D99 of the cloud decrease.

As shown in [5], an experiment was conducted on a cloud from which the lower cloud of a two-layer cloud that was relatively affected by the adjacent effect was removed. Fig. 7 shows that in the range of 3 to $4 \mathrm{~km}$ from the edge of the cloud, the adjacent effect is larger in the case of the cloud phase function than in the case of the aerosol phase function, and the ToA radiance is higher. This is thought to be due to the superior forward scattering of the phase function of the cloud particles than that of the aerosol. However, it turns out that there is almost no change with D99. Also, the shadow of the cloud appears at a distance as shown in Fig. 7 because the sun zenith angle is set at 30 degrees, and the brightness is the same in both cases.

The adjacent effect of the cloud is that the contribution of multiple scattered radiation in the cloud is large, so when the forward scattering of the phase function increases, the brightness near the cloud edge increases. It is found that the phase function of cloud droplets affects the adjacent effect [18].

\section{B. Monte Carlo Simulation in the Presence of Clouds in a Layered Atmosphere}

The real atmosphere is a non-uniform atmosphere in the altitude direction. Therefore, in order to assume a model close to reality, the three-dimensional atmospheric cell is made into a layer structure. This time, the atmosphere was divided by using Gauss-Seidel. Gauss-Seidel is a radiative transfer code based on a parallel plate atmospheric model using an iterative convergence calculation [5], [6]. The optical thickness is used to divide the atmosphere into several layers and calculate the radiative transfer. For an optical thickness of 0.2 (aerosol 0.1, air molecule 0.1), Gauss-Seidel divided the atmosphere into 10 layers. Table III shows the altitude and the optical thickness of aerosol and air molecules. For an optical thickness of 0.4 (aerosol 0.2, air molecule 0.2), the atmosphere was divided into 21 layers. Table IV shows the altitude and each optical thickness. Here, clouds were placed in these two opticalthickness layered atmospheric cells, and the effects of cloud adjacency were evaluated by the Monte Carlo method, which was more realistic.

TABLE. II. ToA RADIANCE AND D99 OF THE DIFFERENT PHASE FunCTION OF AEROSOL AND CLOUd PARTICLE

\begin{tabular}{|l|l|l|}
\hline Phase function & ToA Radiance & D99 \\
\hline Aerosol & $1.099 \mathrm{E}-05$ & $7.6 \mathrm{~km}$ \\
\hline Cloud particle & $1.086 \mathrm{E}-05$ & $7.6 \mathrm{~km}$ \\
\hline
\end{tabular}


TABLE. III. OPTICAL DEPTH OF AEROSOL AND MOLECULE FOR TEN LAYERED ATMOSPHERE MODEL

\begin{tabular}{|l|l|l|l|}
\hline Layer & Altitude $(\mathrm{km})$ & Aerosol & Molecule \\
\hline 10th & $14.868 \sim 50.0$ & 0.007325 & 0.011809 \\
\hline 9th & $9.868 \sim 14.868$ & 0.006168 & 0.013963 \\
\hline 8th & $6.668 \sim 9.868$ & 0.00441 & 0.015632 \\
\hline 7th & $4.328 \sim 6.668$ & 0.004286 & 0.0158 \\
\hline 6tyh & $2.708 \sim 4.328$ & 0.006514 & 0.013617 \\
\hline 5th & $1.723 \sim 2.708$ & 0.010541 & 0.009586 \\
\hline 4th & $1.089 \sim 1.723$ & 0.013244 & 0.006767 \\
\hline 3rd & $0.663 \sim 1.089$ & 0.014925 & 0.005178 \\
\hline 2nd & $0.282 \sim 0.663$ & 0.015966 & 0.004171 \\
\hline 1st & $0.0 \sim 0.282$ & 0.016615 & 0.003473 \\
\hline
\end{tabular}

TABLE. IV. OPtical DePth of AEROSOl AND Molecule For 21 LAYERED ATMOSPHERE MODEL

\begin{tabular}{|l|l|l|l|}
\hline Layer & Altitude $(\mathrm{km})$ & Aerosol & Molecule \\
\hline 21 st & $20.018 \sim 50.0$ & 0.004726 & 0.010445 \\
\hline 20th & $15.568 \sim 20.018$ & 0.008392 & 0.010709 \\
\hline 19th & $12.618 \sim 15.568$ & 0.006839 & 0.012443 \\
\hline 18 th & $10.418 \sim 12.618$ & 0.005588 & 0.01373 \\
\hline 17th & $8.668 \sim 10.418$ & 0.004682 & 0.014532 \\
\hline 16th & $7.188 \sim 8.668$ & 0.004138 & 0.01501 \\
\hline 15th & $5.888 \sim 7.188$ & 0.003708 & 0.015549 \\
\hline 14th & $4.788 \sim 5.888$ & 0.004119 & 0.0151 \\
\hline 13th & $3.848 \sim 4.788$ & 0.004819 & 0.014463 \\
\hline 12th & $3.068 \sim 3.848$ & 0.006196 & 0.013183 \\
\hline 11th & $2.458 \sim 3.068$ & 0.007987 & 0.011137 \\
\hline 10th & $1.978 \sim 2.458$ & 0.010032 & 0.009337 \\
\hline 9th & $1.596 \sim 1.978$ & 0.011502 & 0.00708 \\
\hline 8th & $1.284 \sim 1.596$ & 0.012556 & 0.006634 \\
\hline 7th & $1.023 \sim 1.284$ & 0.013349 & 0.005733 \\
\hline 6tyh & $0.798 \sim 1.023$ & 0.014082 & 0.005081 \\
\hline 5th & $0.6 \sim 0.798$ & 0.014738 & 0.004579 \\
\hline 4th & $0.426 \sim 0.6$ & 0.015085 & 0.004109 \\
\hline 3rd & $0.27 \sim 0.426$ & 0.015485 & 0.003754 \\
\hline 2nd & $0.129 \sim 0.27$ & 0.015809 & 0.00345 \\
\hline 1st & $0.0 \sim 0.129$ & 0.016158 & 0.003205 \\
\hline
\end{tabular}

\section{Method for Calculation of the Parameters in the} Structured Atmosphere

The method of determining the photon travel distance at the boundary of layer structure with different optical properties cannot be used in the uniform atmosphere [16]. Therefore, when a photon enters an atmospheric layer with different optical properties, the photon is temporarily stopped at the boundary, and the traveling distance is obtained by a method of newly determining the traveling distance based on the optical parameters of the atmospheric layer newly entering at the boundary [17]. This method is shown in Fig. 8.
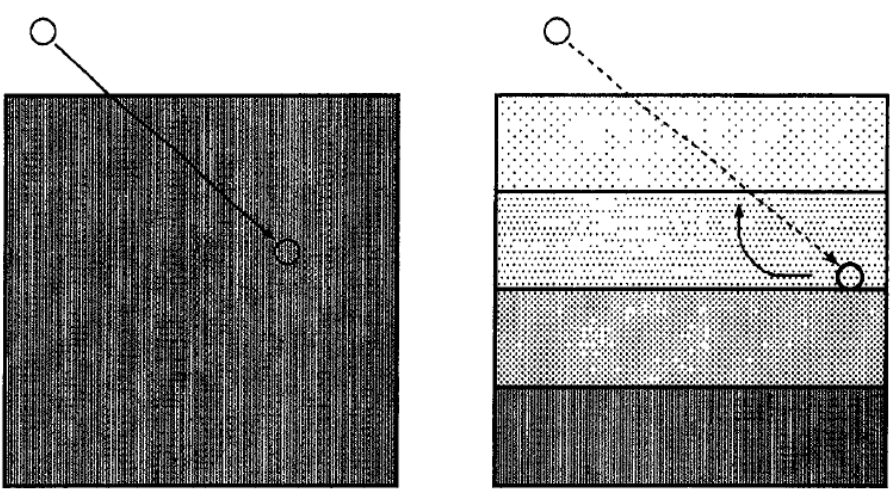

Fig. 8. The Method for Determination of the Final Destination of Photon when the Photons Across the Border of the different Layers.

\section{Limitation of the Proposed Simulation}

There is no limitation of the number of layers, cloud types as well as shape of the clouds for the proposed simulation method. It, however, is difficult to modelized nimbostratus due to the fact that physical characteristics of the nimbostratus is still unclear.

\section{E. Adjacent effects when a Single Cloud exists in the 10- Layer Atmosphere}

Fig. 9 and Table $\mathrm{V}$ show the adjacent effects when a single cloud exists in the 10-layer atmosphere.

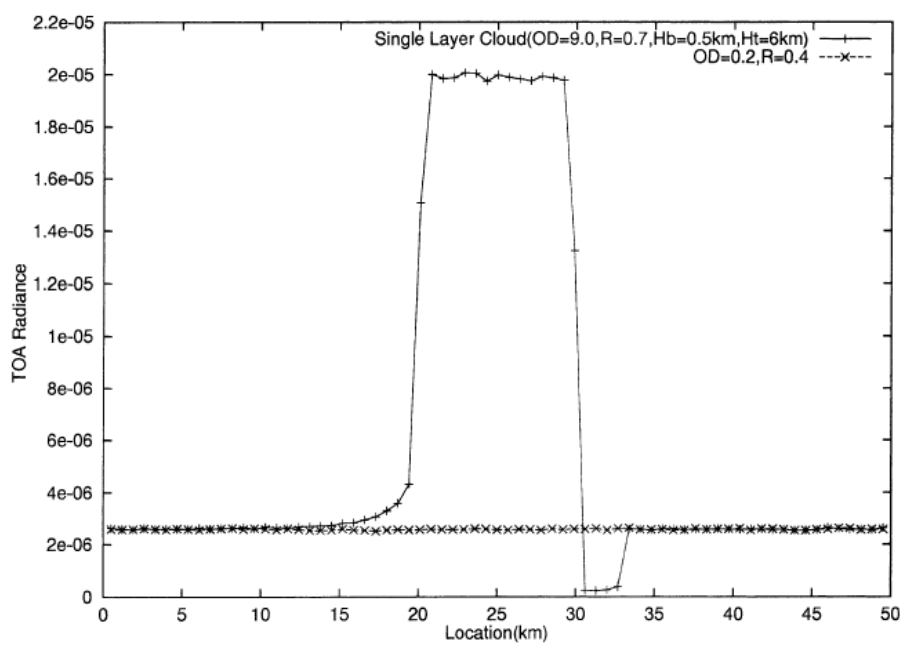

Fig. 9. Adjacent Effects when a Single Cloud Exists in the 10-Layer Atmosphere (A Comparison of the ToA Radiance for the Single and Ten Layered Atmosphere (the unit of ToA Radiance is $\mathrm{W} / \mathrm{cm}^{2} / \mathrm{str} /$ Micrometer)).

TABLE. V. A COMPARISON OF THE MEAN TOA RADIANCE AND D99 FOR THE SINGLE LAYERED CLOUD BETWEEN THE SINGLE AND THE 10 LAYERED ATMOSPHERE

\begin{tabular}{|l|l|l|}
\hline Number of Layer & ToA Radiance & D99 \\
\hline 10 & $1.91 \mathrm{E}-05$ & $5.5 \mathrm{~km}$ \\
\hline 1 & $1.54 \mathrm{E}-05$ & $4.8 \mathrm{~km}$ \\
\hline
\end{tabular}

Both the D99 and the average radiance of the cloud are larger in the one-layer cloud in the ten-layer atmosphere than in the one-layer cloud in the uniform atmosphere. Fig. 10, Table VI shows the adjacent effect when a single cloud exists in the 21 layer atmosphere. Similar to the 10-layer atmosphere, 
the one-layer cloud of the 21-layer atmosphere has a higher D99 and average cloud radiance. In the uniform atmosphere without clouds and the layered atmosphere, the top edge brightness of the atmosphere did not change much even when the reflectance of the ground surface was changed. There was a considerable difference between the effect and the average radiance of the clouds. This is because the upper surface of the cloud is in the 10th layer atmosphere, the 7th layer in the 10th layer atmosphere, and the 15th layer in the 21st layer atmosphere, and the 15th layer and the upper layer of the atmosphere. The actual atmosphere is considered to have a layered structure, and in order to investigate the influence of the adjacent effect more accurately, it is necessary to evaluate the adjacent effect in the layered atmosphere.

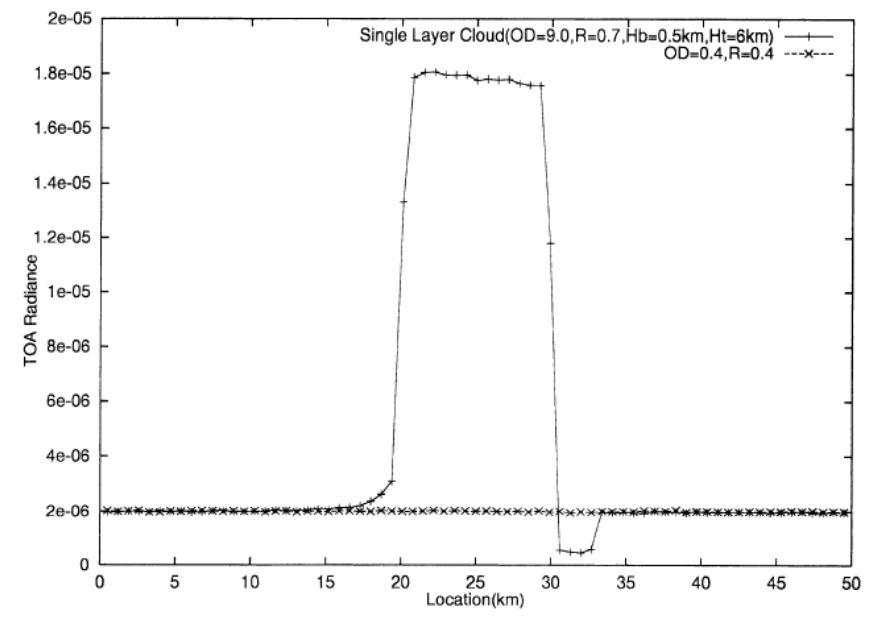

Fig. 10. Adjacency Effect of the Single Layered Cloud for the 21 Layered Atmosphere (the unit of ToA Radiance is $\mathrm{W} / \mathrm{cm}^{2} / \mathrm{str} /$ micrometer).

TABLE. VI. TOA RADIANCE AND D99 FOR THE Single LAYERED Cloud WITH THE SINGLE AND 21 LAYERED ATMOSPHERE

\begin{tabular}{|l|l|l|}
\hline Number of Layer & ToA Radiance & D99 \\
\hline 21 & $1.71 \mathrm{E}-05$ & $3.4 \mathrm{~km}$ \\
\hline 1 & $1.35 \mathrm{E}-05$ & $2.7 \mathrm{~km}$ \\
\hline
\end{tabular}

\section{CONCLUSION}

The adjacent effect of the cloud has a large contribution of radiation scattered in the cloud, so when the forward scattering of the phase function increases, the brightness near the cloud edge increases. This shows that the phase function of cloud particles needs to be considered when evaluating the cloud adjacency effect. In addition, from the evaluation results of the cloud adjacency effect when the number of layers is changed when the atmosphere is considered as a multilayer parallel plate model, it is confirmed that the adjacency effect increases as the number of layers increases.

In other words, since the adjacent effect is caused by multiple scattering in the atmosphere, the multilayer atmosphere is larger than the single layer, and an evaluation close to reality can be expected. This indicates that it is necessary to make the number of layers in the parallel plate atmosphere sufficiently large when evaluating the cloud adjacency effect.

\section{FUTURE RESEARCH WORKS}

Further experimental studies are required for the validation of the proposed method. Also, applicability of the proposed method has to be confirmed through further experiments.

\section{ACKNOWLEDGMENT}

The author would like to thank Prof. Dr. Hiroshi Okumura for his valuable discussions.

\section{REFERENCES}

[1] Kawata, Y., Y. Haba, T. Kusaka, S. Ueno and Y. Terashita, Atmopsheric effects and their cor-rection in airborne sensor and Landsat MSS data,cProceedings of the 12th International Symposium on Remote Sensing of Environment, ERIM, pp.1241-1257, 1978.

[2] Lenoble, J., Edt., Radiometric transfer in scattering and absorbing atmospheres: Standard computational procedures, A Deepack, Publishing 1985.

[3] Tsutomu Takashima and Kazuhiko Masuda, Algorithm for calculating the influence of land reflected light on the radiance from the top of the atmosphere in coastal areas, Journal of the Remote Sensing Society of Japan, Vol.17, No.4, pp.3-13, 1997.

[4] Wilson A., Atmospheric correction of SeaWiFS data: An algorithm and validation for case-2 waters, NASA Technical Memorandom 104566, 31, 1996.

[5] Kobayashi, T., K. Masuda and M. Sasaki, Effects od adjacent clouds to satellite field of view on the measurements of the surface refclectivity, Proceedings of the International Symposium on the Atmospheric Correction of Satellite Data and Its Application to Global Environment, 157-160, 1998.

[6] Kohei Arai and Shinya Kawaguchi, Evaluation of peripheral effects considering cloud layer structure by Monte Carlo method, Journal of Remote Sensing Society of Japan, Vol.21, No.2, pp.179-185, 2001.

[7] Arai, K., Terayama, K., Evaluation of the effects of the peripheral effects of clouds with a layer structure based on the Monte Carlo method, Chiba University Research Center for Environmental Remote Sensing, No.6, pp.52-55, 2000.

[8] Kawada, T., Evaluation of the influence of surrounding clouds on target observation brightness by Monte Carlo method, No. 6, pp. 29-33, 2000.

[9] Kohei Arai, Adjacency effect of layered clouds estimated with MonteCarlo simulation, Advances in Space Research, Vol.29, No.19, 18071812, 2002.

[10] Kohei Arai, Masanori Sakashita, Evaluation of Cirrus Cloud Detection Accuracy of GOSAT/CAI and Landsat-8 with laser Radar: Lidar and Confirmation with Calipso Data, International Journal of Advanced Research on Artificial Intelligence, 5, 1, 14-21, 2016.

[11] Kohei Arai, Masanori Sakashita, Hiroshi okumura, Shuichi Kawakami, Kei Shiomi, Hirofumi Ohyama, Comparative Study on Cloud Parameter Estimation AmongGOSAT/CAI, MODIS, CALIPSO/CALIOP and Landsat-8/OLI with Laser Radar as Truth Data, International Journal of Advanced Research on Artificial Intelligence, 5, 5, 21-29, 2016.

[12] Kohei Arai, Thresholding Based Method for Rain, Cloud Detection with NOAA/AVHRR Data by Means of Jacobi Itteration Method, International Journal of Advanced Research on Artificial Intelligence, 5, 6, 21-27, 2016.

[13] Kohei Arai, Shinya Kawaguchi, Elucidation of adjacent effects of multilayer clouds based on Monte Carlo method, The 30th Annual Conference of the Remote Sensing Society of Japan, B8, pp.49-50, 2001.

[14] WE. Meador, W.R. Weaver, Two-Stream Approximations to Radiative Transfer in Planetary Atmospheres: A Unified Description of Existing Methods and a New Improvement, Journal of Atmospheric Science, 37, 1980.

[15] Tatsushi Tokioka, Masaki Yamasaki, Nobuo Sato, Numerical simulation of meteorology (Meteorological Class 5). The University of Tokyo Press, 247 pp., 1993.

[16] US Air Force Geophysical Laboratory, MODTRAN 3 User Instructions, GL-TR-89-0122, 1996. 
[17] Tomio Asai, Takeda Ikuo, Kimura Ryuji: "Atmosphere Science Course 2 Atmosphere with Clouds and Precipitation" (University of Tokyo Press, p.93., 1981.

[18] Arai Kohei, Basic Theory of Remote Sensing, Academic Book Publishing, 2001.

\section{AUTHOR's PROFILE}

Kohei Arai, He received BS, MS and PhD degrees in 1972, 1974 and 1982, respectively. He was with The Institute for Industrial Science and Technology of the University of Tokyo from April 1974 to December 1978 also was with National Space Development Agency of Japan from January, 1979 to March, 1990. During from 1985 to 1987, he was with Canada Centre for Remote Sensing as a Post Doctoral Fellow of National Science and
Engineering Research Council of Canada. He moved to Saga University as a Professor in Department of Information Science on April 1990. He was a councilor for the Aeronautics and Space related to the Technology Committee of the Ministry of Science and Technology during from 1998 to 2000. He was a councilor of Saga University for 2002 and 2003. He also was an executive councilor for the Remote Sensing Society of Japan for 2003 to 2005. He is an Adjunct Professor of University of Arizona, USA since 1998. He also is Vice Chairman of the Science Commission "A" of ICSU/COSPAR since 2008 then he is now award committee member of ICSU/COSPAR. He wrote 37 books and published 570 journal papers. He received 30 of awards including ICSU/COSPAR Vikram Sarabhai Medal in 2016, and Science award of Ministry of Mister of Education of Japan in 2015. He is now Editor-in-Chief of IJACSA and IJISA. http://teagis.ip.is.saga-u.ac.jp/index.html. 